\title{
Facteurs associés à la survenue d'une épidémie de rougeole : Cas du district de Bamako en 2014
}

\section{Factors associated with the occurrence of a measles epidemic: Case of the district of Bamako in 2014}

\author{
Diarra Moussa Modibo ${ }^{1 *}$, Sangho Oumar², Diallo Oumar ${ }^{3}$, Touré Fady ${ }^{4}$, Koné Abdoulaye ${ }^{5}$, Djourté Guédjouma ${ }^{6}$, Kayentao Kassim²,
} Sangho Fanta ${ }^{7}$, Sangho Hamadoun ${ }^{2}$, Sacko Massambou ${ }^{3}$

1 Centre de santé de référence de Kéniéba

2 Département d'Etude et de Recherche en Santé Publique et spécialité (DERSP)

3 Centre de santé de référence de Ségou

4 Programme National de Lutte contre le Paludisme (PNLP)

5 Direction régionale de la santé de Sikasso

6 Direction Générale de la Santé et de l'Hygiène publique (DGSHP)

7 Direction de la Pharmacie et du Médicament (DPM)

8 Organisation Mondiale de Santé (OMS)

*Auteur correspondant : Moussa Modibo DIARRA MD, MPH Epidémiology ; Médecin Chef du district sanitaire de Kéniéba Tel: 7636 3196 / 66563591 Email: moussamdiarra@gmail.com, moussamdiarra@outlook.fr, toujourfr2002@yahoo.fr

\section{Résumé}

Introduction : L'OMS estime que 145700 personnes, dont une majorité d'enfants de moins de 5 ans, sont mortes de la rougeole en 2013. Une flambée de rougeole a éclaté à Bamako en 2014, faisant 109 cas. Nous nous sommes alors proposé d'analyser les facteurs liés à cette épidémie afin de comprendre leur niveau d'association à celle-ci et de fournir des pistes aux autorités pour la prise des mesures de prévention ciblées. Méthodologie : ॥ s'agissait d'une étude cas-témoin. Les cas constituaient tous les cas confirmés de rougeole à Bamako en 2014 et les témoins tous les cas suspects de rougeole mais non confirmés et les cas notifiés d'autres maladies épidémiques en 2014 à Bamako. Notre échantillon était constitué de 96 sujets sélectionnés selon un échantillonnage exhaustif pour les cas (48) et aléatoire simple pour les témoins (48). Les données ont été analysées par le logiciel SPSS version 20. Résultats : L'analyse montre que le risque de rougeole était plus élevé chez les non vaccinés OR = 2,55 [1,12-5,82]. Le risque n'a pas été significatif pour les sujets ayant un contact avec un malade OR $=5,77[0,67-49,61]$. Les participants n'ayant pas eu la vitamine $A$ ont un risque plus élevé $\mathrm{OR}=2,83[1,20-6,65]$. Conclusion : la rougeole est influencée par le statut vaccinal et l'administration de la vitamine $A$. Par conséquent les autorités doivent, en tenant compte de ces résultats, coupler la vaccination contre la rougeole à l'administration de la vitamine $A$.

Mots clés : épidémie ; rougeole ; facteurs ; cas - témoins.

\footnotetext{
Abstract

Introduction : WHO estimates that 145700 persons, among them the majority of children under 5 years, died by measles in 2013. A measles explosed in Bamako in 2014, making 109 cases. So we have decided to analyse the factors linked to this epidemic, in order to understand their level of association in this one and to provide the authorities some way in order to take decisions to prevent them. Method : It was a case-control study. The cases
}

constitute all confirmed cases of measles in Bamako in 2014 and the control of all cases suspected unconfirmed of measles and cases notified of other epidemic diseases in 2014 in Bamako. Our study took place in the district of Bamako and concerned a sample of 96 subjects. The sampling was exhaustive for the cases (48) and a simple random for the control (48). The data were analyzed by the software SPSS version 20. Results : Several analyse shows that the risk of measles was higher among the unvaccinated in comparison with those who have been vaccinated $O R=2,55[1,12-5,82]$. The subjects having a contact with a sick person seemed to have more risk OR $=5,77[0,67 ; 49,61]$. The participants having had no vitamin A have a higher risk with compared with those who did not have it $\mathrm{OR}=2,83[1,20 ; 6,65]$. Conclusion : The measles is influenced by the vaccinal status and the administration of the vitamin $A$. Consequently the authorities must take into account these connected results by using both vaccination against measles and vitamin $A$ together.

Keywords : epidemic ; measles ; factors ; case-control.

\section{INTRODUCTION}

La rougeole est une maladie virale aigue extrêmement contagieuse. Elle est due à un virus de la famille des Paramyxoviridae. Le virus rougeoleux se transmet par contact direct ou par l'air. La rougeole est une maladie humaine et touche principalement les enfants $(6$ mois à 14 ans). Les cas adultes sont possibles (1-3).

La rougeole reste un problème de santé publique surtout dans les pays en développement malgré la disponibilité d'un vaccin sûr et efficace. En 1980, avant la généralisation de la vaccination, on estimait à 2,6 millions par an le nombre de décès dus à la rougeole (1). La vaccination contre la rougeole a permis d'éviter 15,6 millions de décès de 2000 à 2013 (3).

Selon l'OMS la rougeole reste l'une des causes importantes de décès du jeune enfant. Elle estime que 145700 personnes, dont une majorité d'enfants de moins de 5 ans, sont mortes de la rougeole en 2013 (1). Les décès sont surtout dus aux complications de la maladie. Celles-ci sont plus fréquentes avant l'âge de 5 ans ou chez l'adulte de plus de 20 ans. Parmi les complications les plus graves, on observe des cas de cécité, d'encéphalite (qui peuvent s'accompagner d'œdèmes cérébraux), de diarrhée sévère (susceptibles d'entraîner une déshydratation), d'infections auriculaires et d'infections respiratoires graves comme la pneumonie (1)

Les formes sévères sont surtout observées chez les jeunes enfants malnutris (apports en vitamine A 
insuffisants) ou s'il y a une immunodépression (VIH/sida) ou d'autres maladies.

Dans les populations fortement touchées par la malnutrition et qui ne bénéficient pas de soins de santé adéquats, jusqu'à $10 \%$ des cas de rougeole sont mortels. Les personnes qui guérissent de la rougeole sont immunisées à vie (1).

En Afrique, la rougeole est la quatrième cause majeure de mortalité chez les enfants de moins de 5 ans dans les pays en développement (4).

Le Mali a connu plusieurs flambées épidémiques de rougeole (1998, 1999, 2001, 2008, 2009, 2010, 2011, $2012,2013)(5)$. La dernière grosse épidémie de rougeole remonte à 1998 avec plus de 8009 cas enregistrés dont 3362 pour la capitale Bamako. Si cette fréquence est élevée, la létalité cependant reste à 1\% (5). En 2012, vingt un districts ont connu l'épidémie de rougeole et dix-neuf entre mars et juin (5).

La rougeole est sur la liste des maladies à déclaration obligatoire (MADO) surveillées au Mali (4). La surveillance de ses maladies se fait quotidiennement et suit la pyramide sanitaire. Le Mali a élaboré un plan stratégique national d'élimination de la rougeole (2013-2020), son but est de parvenir à l'élimination de la rougeole au Mali d'ici fin 2020 (6). La vaccination anti rougeole (routine et campagne de masse) à travers la mise en place du Programme Elargi de Vaccination (PEV) a permis une réduction de la mortalité et de la morbidité de la rougeole de $98 \%$ (1), la supplémentation nutritionnelle (vitamine A, produits alimentaires) et les mesures d'hygiène sont entre autres les mesures de prévention de la survenue d'une épidémie de rougeole au Mali. Malgré ces dispositions, une flambé de rougeole a éclaté à Bamako en 2014 faisant 109 Cas (suspects et confirmés) mais sans décès (7). Plusieurs facteurs peuvent entrer en ligne de compte dans la survenue de cette épidémie se sont entre autres :

- $\quad$ La promiscuité des enfants réceptifs

- Les mauvaises conditions d'hygiène

- L'âge précoce, moins de 2ans (essentiellement en milieu urbain)

- La malnutrition

- Les maladies débilitantes (VIH, tuberculose, cancer, etc...)

- Les conditions socioéconomiques précaires (pauvreté)

- $\quad$ La faible couverture vaccinale $(<80 \%)$

- Les mouvements des populations.

Des études ont été menées au Mali et ailleurs, mais toutes étaient essentiellement des études descriptives. C'est devant cette situation que nous nous sommes proposés de mener la présente étude afin d'analyser les facteurs liés à la survenue de cette épidémie. Cette étude pourra permettre de mieux comprendre le niveau d'association des facteurs liés à la survenue d'une épidémie de rougeole et par conséquent de prendre des mesures de prévention ciblées.

\section{METHODOLOGIE}

Type d'étude :

II s'agissait d'une étude cas-témoins qui a consisté à choisir les cas parmi les sujets chez lesquels la rougeole a été confirmé à Bamako en 2014 soit au laboratoire $(\lg \mathrm{M}+)$, soit par le lien avec une épidémie ; et les témoins parmi les suspects de rougeole mais chez qui le test de confirmation au laboratoire a été négatif et le lien avec une épidémie n'est pas établi en 2014 à Bamako mais aussi parmi les autres cas de maladies (méningite, ictère fébrile et PFA(Paralysie Flasque Aigue)) notifiées dans le cadre de la surveillance épidémiologique.

Lieu et période d'étude :

Notre étude s'est déroulée dans le district de Bamako qui s'étend sur 30 km d'Ouest en Est et sur $20 \mathrm{Km}$ du Nord au Sud. II est limité à l'Ouest par la commune du Mandé, au Sud par la commune de Kalaban Coro, au Sud Est par la commune de Baguineda, à l'Est par la commune de Moribabougou et au Nord par la commune de Kati. II couvre une superficie totale d'environ $267 \mathrm{Km}^{2}$ et une population résidente en 2014 de 2156999 habitants (RGPH 2009 actualisé) soit une densité de 8079 habitants $/ \mathrm{km}^{2}(7)$.

Population d'étude :

- Pour les cas : tous les cas confirmés de rougeole à Bamako en 2014.

- Pour les témoins : deux groupes, les cas suspects de rougeole mais non confirmés en 2014 à Bamako et les autres cas de maladies (méningite, ictère fébrile, PFA) notifiés en 2014 à Bamako.

\section{Critères inclusion :}

Ont été inclus dans l'étude tous les cas notifiés de rougeole, de méningite, de ictère fébrile et de PFA ayant une fiche de notification correctement remplie à Bamako en 2014.

Critères de non inclusion :

N'ont pas été inclus dans l'étude, les cas non résidents à Bamako ou en dehors de l'année 2014 et les cas n'ayant pas de fiche de notification ou lorsque celle-ci est incomplètement remplie.

\section{Echantillonnage}

Taille de l'échantillon :

La taille minimum de l'échantillon a été calculée par le programme StatCalc du logiciel " Epi Info 7». Les éléments du calcul sont les suivants :

- $\quad$ Niveau de confiance : $95 \%$

- Puissance de l'étude : $80 \%$

- Ratio témoins - cas : 1

- Pourcentage de témoins exposés (non vaccinés) : $50 \%$

- Pourcentage de non vacciné chez les cas : $80 \%$ (8)

- Odds ratio : 4

La taille minimum reçue est 90 (45 cas et 45 témoins). Technique d'échantillonnage :

- Pour les cas, l'échantillonnage a été non probabiliste (exhaustif). Tous les cas confirmés (IgM+ ou lien 
épidémique) ont été enrôlés dans l'étude soit 48 sujets en 2014.

- Pour les témoins, un échantillonnage aléatoire simple a été utilisé. La base de sondage était une liste (par numéro d'ordre d'enregistrement) exhaustive de tous les cas suspects de rougeole et les cas notifiés de méningite et de ictère fébrile. Nous avons utilisé le logiciel Excel pour sélectionner au hasard 48 sujets dans la liste. Chaque témoin a été apparié à un cas sur l'âge et le sexe.

Nous avons travaillé alors avec une taille d'échantillon de 96 sujets.

\section{Choix des variables}

Variable dépendante :

C'est la rougeole, variable qualitative dichotomique.

Variables indépendantes :

Ce sont :

- l'âge, variable quantitative continue, il a été catégorisé en classe d'âge (inférieur à 1 an, 1 - 4 ans, 5 - 14 ans et 15 ans et plus) ;

- le sexe, variable qualitative dichotomique (masculin et féminin);

- le statut vaccinal, variable qualitative dichotomique (les statuts vaccinaux « inconnu » ont été considérés comme non vacciné) ;

- l'administration de la vitamine A, variable qualitative tri modale (oui, non et inconnu) ;

- $\quad$ la notion de contact (avoir un cas similaire dans les alentours) avec un malade, variable qualitative tri modale (contact, pas de contact et inconnu) ;

- la notion de voyage précédant la maladie, variable qualitative tri modale (oui, non et inconnu) ;

- la résidence, variable qualitative pluri modale (Commune I, II, III, IV, V, VI) ;

Plan pour la collecte des données :

Les données ont été collectées dans les fiches de notification et les rapports d'investigation des cas. Ces données ont été directement saisies dans un masque élaboré sur SPSS 20.

Instruments de collecte des données :

Un ordinateur a été utilisé pour la collecte des données.

Plan pour l'analyse des données :

Les données ont été analysées par le logiciel SPSS version 20. Nous avons procédé à une analyse uni variée (moyenne, minimum, maximum, écart type pour les variables quantitatives et fréquence pour les variables qualitatives, ratio), bi variée (croisement entre la variable dépendante et les variables indépendantes), multi variée (une régression logistique avec l'ensemble des variables indépendantes). Nous avons fait aussi une description de l'épidémie en fonction du temps, du lieu et des individus. Les données analysées ont été présentées sous forme de tableaux et ou de figures appuyés d'un commentaire. Nous avons utilisé l'Odds Ratio, le Chi2 ou le test exact de Ficher pour déterminer le niveau des associations. Le seuil de significativité statistique a été fixé à $5 \%$. Tout $p$ inférieur à ce seuil a été considéré comme significatif.

Problèmes déontologiques :

La présente étude a été réalisée dans le respect strict des codes éthiques et de la déontologie. L'anonymat et la confidentialité ont été gardés. Puisqu'il s'agissait d'une collecte rétrospective, il n'a pas été aucunement possible de relier les informations recueillies aux personnes physiques.

\section{Exploitation des données :}

Les résultats pourront être utilisés par le système de surveillance des maladies à potentiel épidémique, pour renforcer la prévention des épidémies de rougeole.

Diffusion et utilisation des résultats :

Les résultats ont été déposés à la bibliothèque du Département d'Enseignement et de Recherche en Santé Publique (DERSP), à la Direction Régionale de la Santé de Bamako (DRS). Une publication des résultats sera envisagée.

\section{Définitions opératoires}

Epidémie de rougeole : Une épidémie de rougeole est définit par la notification de cinq cas suspects ou la confirmation d'au moins trois cas au laboratoire par mois.

Cas suspect de rougeole : Toute personne présentant de la fièvre, une éruption généralisée maculopapulaire (non vésiculaire) et de la toux ou un rhume ou une conjonctivite (yeux rouges), ou toute personne chez laquelle un médecin soupçonne une rougeole (4).

Cas confirmé de rougeole : tout cas, confirmé en laboratoire (sérologie positive des lgM) ou ayant un lien épidémiologique avec des cas confirmés ou une flambée épidémique (4).

\section{RESULTATS}

\section{Analyse uni variée}

Notre étude a porté sur 96 dossiers de notification entre janvier et décembre 2014. Cette population était à majorité masculin $60,4 \%(58 / 96)$ soit un sex ratio de 1,5.

La tranche d'âge la plus représentée était 12 - 59 mois avec $45,8 \%$ (44/96). Les moins de 12 mois ont constitué $30,2 \%$ (29/96). La moyenne d'âge des sujets était de 40,17 mois avec un écart type de 4,20 et un IC à $95 \%$ de $[31,83 ; 48,52]$. L'âge minimum était de 1 mois et le maximum 168 mois. II n'y avait pas de différence entre la moyenne d'âge des cas et celle des témoins, $p=0,349$. Les districts sanitaires des communes III et IV ont fait plus de notification soit respectivement $30,2 \%$ et $45,8 \%$ (29/96 et 44/96). Plus de la moitié des sujets résidaient en Commune IV $(51,0 \%) ; 21,9 \%$ résidaient en commune III et $11,5 \%$ en commune Vl.

Quant au statut vaccinal, seulement $53,1 \%$ des participants étaient vaccinés contre la rougeole (51/96). Le taux de sujets non vaccinés est plus élevé chez les cas $(58,3 \%)$ que chez les témoins $(35,4 \%)$.

Le contact contagieux était retrouvé chez $30,8 \%$ des participants (16/52). Un prélèvement d'échantillon de sang a été effectué chez $90,9 \%$ (80/88) des sujets pour la confirmation de la rougeole. Les résultats de ses tests de 
confirmation ont été positifs ( $\operatorname{lgM}+$ ) chez 7 participants soit $13,7 \%$ (7/51). Aucun cas de décès n'a été enregistré. Dans notre étude, la notification de rougeole a représenté $77,1 \%$ (74/96), le reste $(22,9 \%)$ les autres maladies notifiées (méningite, ictère fébrile, PFA). Concernant la profession du père, les commerçants étaient les plus représentés $(34,4 \%)$ suivi des salariés et des ouvriers $(21,9 \%$ chacun). Pour la profession de la mère, les ménagères représentaient $88,6 \%$ (39/44) et les salariées $4,5 \%$ (2/44). La vitamine A a été administrée chez 54,9\% de nos participants (50/91). Seulement $42,2 \%$ des cas avaient reçu la vitamine ; ce taux était de $67,4 \%$ pour les témoins. Et 25,8\% (16/62) avaient une notion de voyage précédant la maladie.

Les taux d'attaque de la rougeole les plus élevés ont été observés en commune II 14,31 cas pour 100000 par an et en commune IV avec 11,74 cas pour 100000 par an (tableau IV).

Des notifications ont été faites tous les mois de l'année. Le maximum de notification a été observé en mars $(20,8 \%)$ et en février $(13,5 \%)$. La moyenne de notification mensuelle est 5,75 avec un écart type de 3,18. La presque totalité de ces cas (6/7) résidaient en commune IV et 1 cas en commune III.

\section{Analyse bi variée}

II existe une association statistiquement significative entre la rougeole et les variables résidence, statut vaccinal et vitamine $A$. Les autres variables ont une association non statistiquement significative.

II n'y avait pas de différence entre les moins de 12 mois et les autres tranches d'âges ( $12-59$ mois et $60-179$ mois) respectivement $\mathrm{OR}=1,34[0,45-4,04]$ et $0,99[0,36-$ $2,73]$. Le risque de rougeole était plus élevé chez les non vaccinés par rapport aux sujets vaccinés $O R=2,55[1,12$ - 5,82]. Les sujets ayant un contact avec un malade semblait avoir plus de risque $\mathrm{OR}=5,77[0,67-49,61]$. Les participants n'ayant pas eu la vitamine $A$ ont un risque plus élevé par rapport à ceux qui n'en ont pas eu $\mathrm{OR}=2,83$ $[1,20-6,65]$.

Concernant la notion de déplacement, les participants avec une notion de mobilité précédant la maladie ont une protection non statistiquement significative par rapport à ceux ayant une notion de mobilité $O R=0,62[0,19-1,99]$. De même pour la profession des parents, les enfants des non-salariés ont une protection non significative par rapport aux salariés $\mathrm{OR}=0,15[0,02-1,29]$.

\section{Analyse multivariée}

Les sujets non vaccinés ont un risque de $1,22 p=0,871$ après ajustement sur les caractéristiques âge, vitamine $A$, profession du père et celle de la mère chez les témoins non suspect de rougeole. Les sujets qui n'ont pas reçu la vitamine $A$ ont un risque plus élevé que ceux qui en ont reçu OR ajusté $=3,71[0,26-38,47]$. Les enfants des pères non-salariés ont une protection de $69 \%, p=0,356$ après ajustement sur statut vaccinal, vitamine $A$, âge, profession de la mère chez les témoins suspects de rougeole. De même les enfants des mères non salariées ont un risque plus élevé de rougeole OR ajusté $=2,45$ $[0,05-39,65]$.

\section{DISCUSSION}

Cette étude a analysé les facteurs associés à la rougeole. Elle a concerné 96 dossiers entre janvier et décembre 2014 dans la région sanitaire de Bamako. Soit 48 cas et 48 témoins. Cet effectif est inférieur à celui de Akramuzzaman SM et al. à Dhaka (Bangladesh) en 2002 qui concerné 1466 dossiers (318 cas et 1148 témoins) (9). Dans notre étude les témoins ont été appariés sur l'âge et le sexe alors que dans celle de Akramuzzaman SM. et al. à Dhaka (Bangladesh) en 2002, l'appariement porte sur l'âge et la résidence (9).

Dans notre étude les garçons étaient les plus représentés $(60,4 \%)$. Ce résultat est comparable à celui de Youbi $\mathrm{M}$. en 2012 au Maroc qui trouve une représentativité de $51,3 \%$ (10) ; il est cependant contraire au résultat de Mahamud $A$. où les femmes étaient plus représentées avec $56,2 \%$ (11).

L'âge dans notre population d'étude était compris entre 1 mois et 14 ans avec une moyenne de 3ans environ ; ce qui est différent de l'âge de la population d'étude de Youbi M. en 2012 au Maroc qui s'étend de 1 mois à 70 ans avec une moyenne de 6 ans (10).

Le taux de de rougeole confirmée (IgM positif) était de $13,7 \%$ dans notre étude. Ce taux est inférieur à celui trouvé en 2010 au Mali (68\%) (12) et en France en mars $2011(36,1 \%)(13)$.

Aucun décès n'a été enregistré dans notre étude, contrairement aux statistiques de la DNS où 56,7 et 5 cas ont été enregistrés respectivement en 2005, 2009 et 2010 (12).

Le statut vaccinal occupait une place importante dans l'étude. En effet environ 46,9\% de nos sujets étaient non vaccinés contre la rougeole, ceci est inférieur au résultat de Mahamud $A$. et al. qui trouvent $64,2 \%$ de non vaccinés chez les réfugiés somaliens déplacés en Kenya en 2011 (11). Pinchoff J. et al. trouvent $50 \%$ de non vaccinés entre 1998 et 2002 et $55 \%$ en 2010 en Zambie (14). Le risque de rougeole que nous avons observé chez les non vaccinés était de 2,55 [1,12-5,82]). Ce résultat est comparable à celui de Mahamud $A$. et al. chez les réfugiés somaliens déplacés en Kenya en 2011 (OR=2,2 [5,0 ; 10,2]) (11) mais inférieur à celui de Pomerai KW et al. dans une étude d'investigation d'une épidémie de rougeole à Zaka (Zimbabwe) en 2010 (OR = 12,46 [6,20; 25,31]) (15).

La vitamine A n'a pas été administrée chez 45,05 \% de nos sujets, ce qui est proche de ce que Mahamud $A$. et al. trouvent $(49,22 \%)$ chez les réfugiés somaliens déplacés en Kenya en 2011 (11). La non prise de vitamine A est apparue comme facteur associé à la survenue de la rougeole avec un Odd Ratio de 2,83 [1,20 - 6,65] ce qui est contraire aux résultats de Mahamud $A$. et al. qui trouvent les OR de $1,1(0,3-3,2)$ et $1(0,3-3,4)$ respectivement pour moins de 2 doses et plus de 2 doses 
de vitamines chez les réfugiés somaliens déplacés en Kenya en 2011 (11).

Le contact avec un malade retrouvé chez $30,8 \%$ de nos participants est inférieur à la fréquence retrouvée par Pomerai K. W. et al., 52,7\% dans une étude d'investigation d'une épidémie de rougeole à Zaka (Zimbabwe) en 2010. Le risque de rougeole lié au contact avec un malade était non significatif $(\mathrm{OR}=5,77[0,67-49,61])$, contrairement à celui trouvé par Pomerai K. W. et al. dans une étude d'investigation d'une épidémie de rougeole à Zaka (Zimbabwe) en 2010 (OR = 169 [57.73-499.78]) (15) .

Concernant la profession de la mère,, les résultats obtenus dans notre étude $(86,4 \%$ de ménagère) sont assimilable à ceux de Akramuzzaman S. M. et al. (respectivement $80 \%$ et $84 \%$ de ménagère pour les cas et les témoins) à Dhaka (Bangladesh) en 2002 (9). Ce facteur, dans notre étude constitue un risque non significatif $(p=1,00)$; ce constat est le même dans l'étude de Akramuzzaman S. M. et al. à Dhaka (Bangladesh) en 2002 (OR=1,1 [0,5-4,0]).

La non signification de l'influence de la vitamine $A$ et du statut vaccinal dans l'analyse multi variée pourrait s'expliquer par l'effet des autres facteurs et la taille de l'échantillon.

\section{CONCLUSION}

Notre étude avait pour but d'analyser les facteurs associés à une épidémie de rougeole à Bamako. La revue de la littérature nous a permis d'identifier et sélectionner les différents facteurs associés à la rougeole qui ont été pris comme variables. Nous avons formulé des hypothèses que nous avons vérifiées par la suite en utilisant les tests statistiques. A travers ces résultats, les autorités doivent augmenter la couverture vaccinale en rougeole et coupler la vaccination contre la rougeole à l'administration de la vitamine A. Cette étude rétrospective n'a pas analysé tous les facteurs associés à la rougeole compte tenu de la disponibilité des informations. Alors une étude analytique prospective serait utile afin de prendre en compte plus de facteurs.

\section{REFERENCES}

1. OMS. Rougeole. Aide-mémoire. OMS, 2015 2015/02//. Report No.: 286.

2. Danet C, Fermon F. Prise en charge d'une épidémie de rougeole. Médecins Sans Frontières ed: Catherine Bachy; Véronique Grouzard; 2013 2013. 240 p. 3. rougeole [Internet]. [cité 5 juill 2015]. Disponible sur: http://medecinetropicale.free.fr/cours/rougeole.pdf.

4. OMS, CDC, USAID, Mali M. Guide Technique pour la Surveillance Intégrée de la Maladie et la Riposte au Mali. 2012.

5. TOUNKARA BS, M. . Analyse de risque d'épidémie rougeole au Mali. p. 18.

6. Bulletin d'information des activités du cluster santé du Mali.29:6.
7. DRS B. Canevas de synthese des rapports d'evaluation 2014 et de programmation 2016 niveau region district de bamako. Bamako: DRS, 20152015. Report No.

8. Zahidie A, Wasim S, Fatmi Z. Vaccine effectiveness and risk factors associated with measles among children presenting to the hospitals of karachi, pakistan. Journal of the College of Physicians and Surgeons-Pakistan: JCPSP. 2014;24(12):882-8.

9. Syed M. Akramuzzaman DI, Felicity T. Cutts, Narayan C. Shaha, Md J. Hossain, Dilip Mahalanabis, Obaidullah K. Wahedi, Nazmun Nahar. Measles vaccine effectiveness and risk factors for measles in Dhaka, Bangladesh. Bulletin of the World Health Organization. 2002;80.

10. Youbi M. FACTEURS PREDICTIFS DE LA ROUGEOLE EN CAS DE FIEVRE ERUPTIVE. Rapport. FETP- MOROCCO, 2012.

11. Mahamud A, Burton A, Hassan M, Ahmed JA, Wagacha JB, Spiegel $P$, et al. Risk factors for measles mortality among hospitalized Somali refugees displaced by famine, Kenya, 2011. Clinical infectious diseases : an official publication of the Infectious Diseases Society of America. 2013;57(8):e160-6.

12. Plan Pluriannuel Complet 2012-2016 [Internet]. [cité 6 janv 2016]. Disponible sur: http://www.gavi.org/Country/Mali/Documents/CMYPs/Co mprehensive-multi-year-plan-for-2012-2016/.

13. «l'épidémie de rougeole explose, entraînant une augmentation des hospitalisations et des formes sévères » Conférence de presse; 23 Mars 20112011. p. 22.

14. Pinchoff J, Chipeta J, Banda GC, Miti S, Shields $\mathrm{T}$, Curriero $\mathrm{F}$, et al. Spatial clustering of measles cases during endemic (1998-2002) and epidemic (2010) periods in Lusaka, Zambia. BMC infectious diseases. 2015;15:121.

15. Pomerai KW, Mudyiradima RF, Gombe NT. Measles outbreak investigation in Zaka, Masvingo Province, Zimbabwe, 2010. BMC research notes. 2012;5(1):687

\section{Liste des tableaux et figures}

Tableau I : Répartition des 96 sujets (cas et témoins) de l'étude dans le district de Bamako en 2014 en fonction du sexe

\begin{tabular}{llll}
\hline Sexe & Cas & Témoins & $\mathbf{N}(\%)$ \\
\hline \hline Féminin & 19 & 19 & $38(39,6)$ \\
Masculin & 29 & 29 & $58(60,4)$ \\
Total & $\mathbf{4 8}$ & $\mathbf{4 8}$ & $\mathbf{9 6}(\mathbf{1 0 0 , 0 )}$ \\
\hline \hline
\end{tabular}

Tableau II : Répartition des 96 sujets (cas et témoins) de l'étude dans le district de Bamako en 2014 en fonction des tranches d'âge

\begin{tabular}{llll}
\hline $\begin{array}{l}\text { Tranches } \\
\text { d'âges }\end{array}$ & Cas & Témoins & N (\%) \\
\hline
\end{tabular}


MALI SANTE PUBLIQUE 2020 SOMASAP

\begin{tabular}{lccc}
\hline \hline Moins de & 16 & 13 & $29(30,2)$ \\
$\begin{array}{l}\text { 12mois } \\
12-59 \text { mois }\end{array}$ & 21 & 23 & $44(45,8)$
\end{tabular}

60 - 179 mois

Total

11

$48 \quad 48$

12

48
$23(24,0)$ $96(100,0)$

Tableau III : Répartition en fonction des caractéristiques individuelles des sujets de l'étude dans le district de Bamako en 2014

\begin{tabular}{|c|c|c|c|}
\hline Caractéristiques & Cas & Témoin & $\mathrm{N}(\%$ \\
\hline \multicolumn{4}{|c|}{ District Sanitaire $(n=96)$} \\
\hline $\mathrm{Cl}$ & 0 & 1 & $1(1,0)$ \\
\hline $\mathrm{Cll}$ & 0 & 10 & $10(10,4)$ \\
\hline $\mathrm{CIII}$ & 6 & 23 & $29(30,2)$ \\
\hline CIV & 42 & 2 & $44(45,8)$ \\
\hline CV & 0 & 3 & $3(3,1)$ \\
\hline CVI & 0 & 9 & $9(9,4)$ \\
\hline \multicolumn{4}{|l|}{ Résidence $(n=96)$} \\
\hline $\mathrm{Cl}$ & 0 & 2 & $2(2,1)$ \\
\hline $\mathrm{Cll}$ & 0 & 9 & $9(9,4)$ \\
\hline $\mathrm{CIII}$ & 1 & 20 & $21(21,9)$ \\
\hline CIV & 47 & 2 & $49(51,0)$ \\
\hline CV & 0 & 4 & $4(4,2)$ \\
\hline CVI & 0 & 11 & $11(11,5)$ \\
\hline \multicolumn{4}{|c|}{ Statut vaccinal $(n=96)$} \\
\hline Non vacciné & 28 & 17 & $45(46,9)$ \\
\hline Vacciné & 20 & 31 & $51(53,1)$ \\
\hline \multicolumn{4}{|c|}{ Contact avec un malade $(n=52)$} \\
\hline Pas de contact & 26 & 10 & $36(69,2)$ \\
\hline Contact & 1 & 15 & $16(30,8)$ \\
\hline \multicolumn{4}{|c|}{ Echantillon prélevé ( $n=88$ ) } \\
\hline Non & 8 & 0 & $8(9,1)$ \\
\hline Oui & 40 & 40 & $80(90,9)$ \\
\hline \multicolumn{4}{|c|}{ Résultat positif $(n=51$ ) } \\
\hline Non & 15 & 29 & $44(86,3)$ \\
\hline Oui & 7 & 0 & $7(13,7)$ \\
\hline \multicolumn{4}{|l|}{ Evolution $(n=96)$} \\
\hline Vivant & 48 & 48 & $96(100,0)$ \\
\hline \multicolumn{4}{|c|}{ Maladies notifiées ( $n=96$ ) } \\
\hline Autres maladies & 0 & 22 & $22(22,9)$ \\
\hline Rougeole & 48 & 26 & $74(77,1)$ \\
\hline \multicolumn{4}{|c|}{ Profession du père $(n=32)$} \\
\hline Salarié & 6 & 1 & $7(21,9)$ \\
\hline Commerçant & 11 & 0 & $11(34,4)$ \\
\hline Ouvrier & 7 & 0 & $7(21,9)$ \\
\hline Libérale & 1 & 0 & $1(3,1)$ \\
\hline Autres & 5 & 1 & $6(18,8)$ \\
\hline \multicolumn{4}{|c|}{ Profession de la mère $(n=44)$} \\
\hline Ménagère & 37 & 2 & $39(88,6)$ \\
\hline Salariée & 1 & 1 & $2(4,5)$ \\
\hline Autres & 3 & 0 & $3(6,8)$ \\
\hline \multicolumn{4}{|l|}{ Vitamine $A(n=91)$} \\
\hline Non & 15 & 26 & $41(45,1)$ \\
\hline Oui & 31 & 19 & $50(54,9)$ \\
\hline \multicolumn{4}{|l|}{ Mobilité ( $n=62$ ) } \\
\hline Non & 15 & 31 & $46(74,2)$ \\
\hline Oui & 7 & 9 & $16(25,8)$ \\
\hline
\end{tabular}


Tableau IV : Association bi variée entre rougeole et les autres variables

\begin{tabular}{|c|c|c|c|c|}
\hline Variables & Témoins [\% (n)] & Cas [\% (n)] & OR (IC à 95\%) & $p$ value \\
\hline \multicolumn{5}{|c|}{ Tranches d'âges en mois } \\
\hline Moins de 12 mois & $27,1(13)$ & $33,3(16)$ & 1,00 (référence) & \\
\hline $12-59$ mois & $47,9(23)$ & $43,8(21)$ & $1,34[0,45-4,04]$ & 0,599 \\
\hline $60-179$ mois & $25,0(12)$ & $22,9(11)$ & $0,99[0,36-2,73]$ & 0,994 \\
\hline \multicolumn{5}{|l|}{ Résidence } \\
\hline $\mathrm{Cl}$ & $4,2(2)$ & $0,0(0)$ & & 0,000 \\
\hline $\mathrm{Cll}$ & $18,8(9)$ & $0,0(0)$ & & \\
\hline CIII & $41,7(20)$ & $2,1(1)$ & & \\
\hline CIV & $4,2(2)$ & $97,9(47)$ & & \\
\hline CV & $8,3(4)$ & $0,0(0)$ & & \\
\hline CVI & $22,9(11)$ & $0,0(0)$ & & \\
\hline \multicolumn{5}{|l|}{ Statut vaccinal } \\
\hline Non vacciné & $35,4(17)$ & $58,3(28)$ & $2,55[1,12-5,82]$ & 0,026 \\
\hline Vacciné & $64,6(31)$ & $41,7(20)$ & 1,00 (référence) & \\
\hline \multicolumn{5}{|c|}{ Contact avec un malade } \\
\hline Pas de contact & $90,9(10)$ & $63,4(26)$ & 1,00 (référence) & \\
\hline Contact & $9,1(1)$ & $36,6(15)$ & $5,77[0,67-49,61]$ & 0,110 \\
\hline \multicolumn{5}{|l|}{ Profession du père } \\
\hline Non salarié & $97,9(47)$ & $87,5(42)$ & $0,15[0,02-1,29]$ & 0,084 \\
\hline Salarié & $2,1(1)$ & $12,5(6)$ & 1,00 (référence) & \\
\hline \multicolumn{5}{|l|}{ Profession de la mère } \\
\hline Non salariée & $97,9(47)$ & $97,9(47)$ & $1,00[0,06-16,46]$ & 1,000 \\
\hline Salariée & $2,1(1)$ & $2,1(1)$ & 1,00 (référence) & \\
\hline \multicolumn{5}{|l|}{ Vitamine A } \\
\hline Non & $32,6(15)$ & $57,8(26)$ & $2,83[1,20-6,65]$ & 0,017 \\
\hline Oui & $67,4(31)$ & $42,2(19)$ & 1,00 (référence) & \\
\hline \multicolumn{5}{|l|}{ Mobilité } \\
\hline Non & $68,2(15)$ & $77,5(31)$ & 1,00 (référence) & \\
\hline Oui & $31,8(7)$ & $22,5(9)$ & $0,62[0,19-1,99]$ & 0,424 \\
\hline
\end{tabular}

Tableau V : Modèle de régression multivariée des facteurs associés à la rougeole à Bamako en 2014 (N = 91)

\begin{tabular}{|c|c|c|c|c|}
\hline Caractéristiques & $\begin{array}{l}\text { Témoin1* } \\
\text { OR ajusté (IC à 95\%) }\end{array}$ & $P$ value & $\begin{array}{l}\text { Témoin2** } \\
\text { OR ajusté (IC à 95\%) }\end{array}$ & $P$ value \\
\hline \multicolumn{5}{|c|}{ Tranches d'âges en mois } \\
\hline Moins de 12 mois & 1,00 (référence) & & & \\
\hline $12-59$ mois & $0,79(0,22-2,87)$ & 0,717 & $1,41[0,36-5,57]$ & 0,624 \\
\hline $60-179$ mois & $1,09(0,21-5,70)$ & 0,917 & $1,24[0,27-5,83]$ & 0,782 \\
\hline \multicolumn{5}{|l|}{ Statut vaccinal } \\
\hline Non vacciné & $0,65(0,03-12,28)$ & 0,773 & $1,29[0,06-27,53]$ & 0,871 \\
\hline Vacciné & 1,00 (référence) & & & \\
\hline \multicolumn{5}{|l|}{ Vitamine A } \\
\hline Non & $2,60(0,15-46,64)$ & 0,516 & $3,71[0,19-72,93]$ & 0,388 \\
\hline Oui & 1,00 (référence) & & & \\
\hline \multicolumn{5}{|l|}{ Profession du père } \\
\hline Non salarié & $0,31(0,03-3,68)$ & 0,356 & & 0,133 \\
\hline Salarié & 1,00 (référence) & & & \\
\hline \multicolumn{5}{|c|}{ Profession de la mère } \\
\hline Non salariée & $2,45(0,94-63,5)$ & 0,591 & & 0,854 \\
\hline Salariée & 1,00 (référence) & & & \\
\hline
\end{tabular}




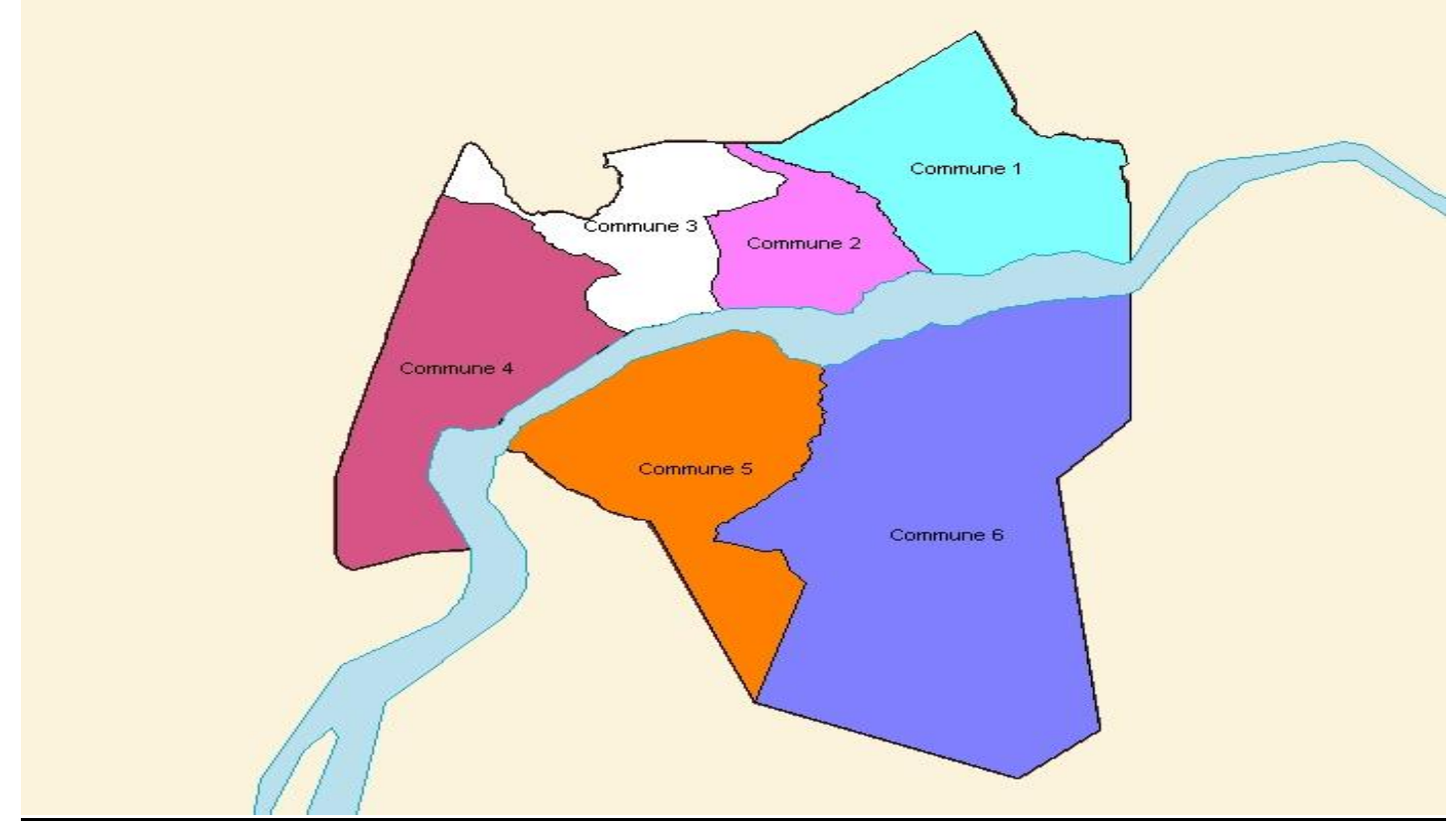

Figure 1 : Carte sanitaire du district de Bamako

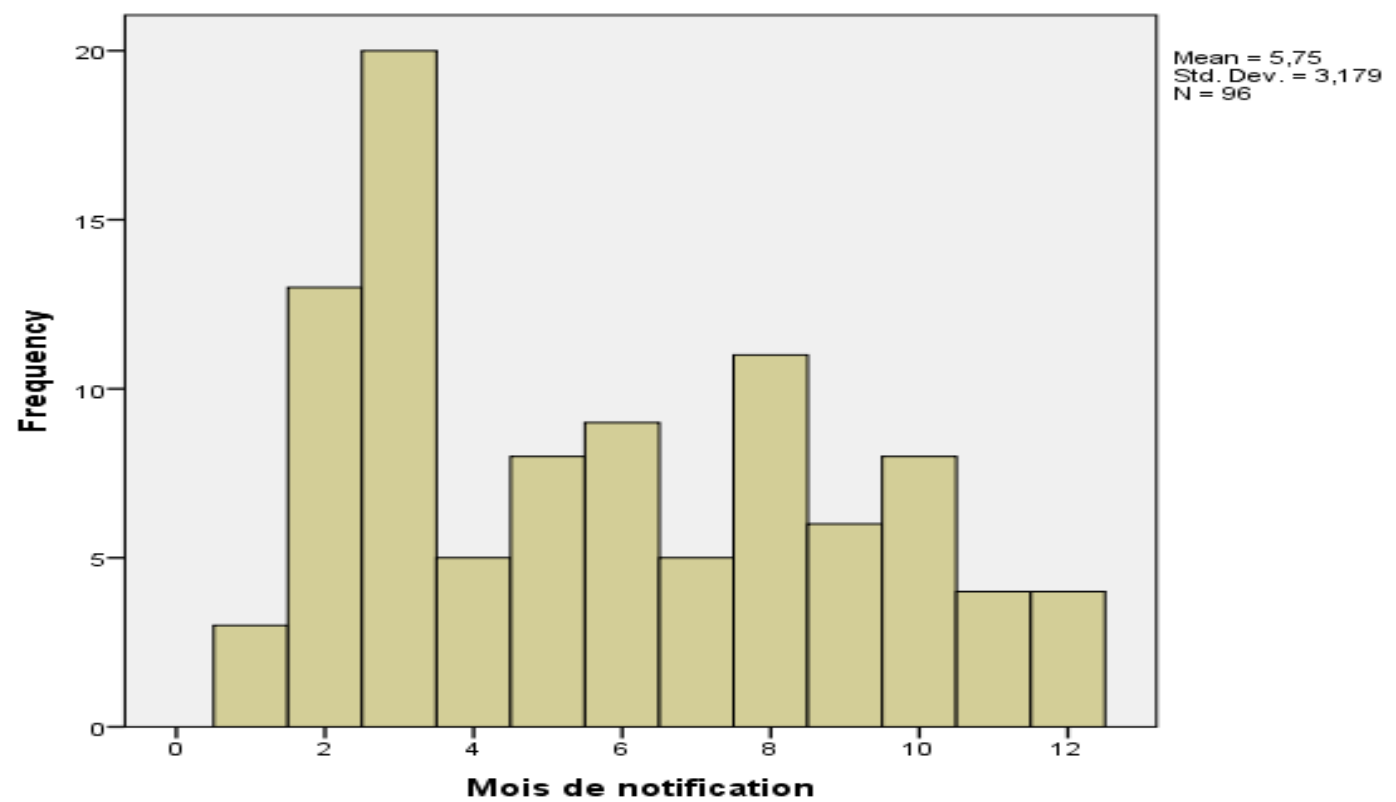

Figure 2: Répartition des sujets de l'étude dans le district de Bamako en 2014 par mois de notification 